\title{
Utility of the chronic unpredictable mild stress model in research on new antidepressants
}

\author{
Karolina Pekala, Barbara Budzynska, Grazyna Biala* \\ Department of Pharmacology and Pharmacodynamics, Medical University of Lublin, Chodzki 4a, 20-093 Lublin, Poland

\begin{tabular}{l}
\hline ARTICLE INFO \\
\hline Received 23 May 2014 \\
Accepted 29 May 2014 \\
\hline
\end{tabular} \\ Keywords: \\ unpredictable chronic mild \\ stress, \\ depression, \\ anhedonia, \\ antidepressants, rodents.

\begin{abstract}
Unpredictable chronic mild stress model was developed as an animal model of depression more than 20 years ago. Essential for this model is that after prolonged exposure of tested animals to a series of unpredictable mild stressors, a condition similar to anhedonia develops, which is observed in the majority of depressive disorders. Unpredictable chronic mild stress model is used nowadays in numerous studies related to the neurobiological and biochemical changes associated with depressive illness. Their results confirm that chronic unpredictable mild stress induces in tested animals a number of changes, which reflect those seen in depressive disorders. Because the effects of unpredictable chronic mild stress can be used in a more accurate diagnosis of the pathophysiology of depressive been continued all the time.
\end{abstract} \\ illness and expand knowledge of its pharmacotherapy, therefore research in this area has
}

\section{INTRODUCTION}

Depression is a severe disorder, which is often manifested by many psychological, behavioral and physiological disturbances. Clinical symptoms characteristic for depression include low self-esteem, fatigue, feelings of worthlessness, loss of interest or pleasure. Nowadays, depression affects $17-20 \%$ of the world population. The World Health Organization (WHO) predicts that depression will be the second leading cause of the global burden of disability in 2020. The etiology of depression is very complicated, and involves a large number of factors [32] including:

- genetic predisposition, which is determined by varying expression of genes related to the specific neurotransmitters,

- recurrent life events and chronic stress,

- specific type of personality development, formed as a result of adverse life experiences, which composes the basis for the internal stress,

- biologically conditioned sensitivity to sunlight deficit (seasonally) [9].

So far, numerous attempts have been made to develop animal models of depression, which allowed focusing on at least some aspects of the disease. These paradigms are

\footnotetext{
Corresponding author

e-mail: grazyna.biala@umlub.pl

tel/fax: +48 81 535-73-71
}

conducted in order to mimic the similarities of clinical symptoms of depression, in laboratory condition. These models make testing many factors involved in the pathogenesis, etiology, symptomatology, and the pharmacological treatment of depression possible [2].

The incidence of depression and its negative consequences still have been intensely studied. The "ideal" animal model of depression should have as many criteria specific to depression in humans as possible, should be sensitive to antidepressants, and should identify similarities in the pathophysiology and etiology of different kind of depressive states [31]. It should be noted that animal models of depression do not always reflect all the irregularities of depression in humans. However, patients do not always reveal all possible symptoms of this disease, either. We may mention here anhedonia, one of the primary symptoms of depression in humans, whereas most of the current animal models of depression only mimic it [9].

\section{MODELS OF DEPRESSION}

Currently, many various models of depression have been used to mimic depressive state in humans. Each model has advantages and disadvantages. The most commonly used paradigms are as follows:

Learned helplessness. In this paradigm the depressive state is induced by uncontrolled and unpredictable electric shock in rodents. Model of learned helplessness was firstly used at the early 60's. In these experiments, animals are 
repeatedly exposed to aversive stimuli without possibility of escape. The animals discontinue attempts to avoid stimuli. As a consequence, when finally possibility to escape becomes available, they do not perform any action [17].

Social defeat stress. This model shows that the majority of stressful stimuli leading to psychopathological changes have a social nature. In this paradigm, it is possible to study the social stress that consequently leads to depressive-like state in rodents [29].

Chronic unpredictable mild stress (CUMS). This model intends to use chronic stress, which leads to the depressive state. The mentioned paradigm was firstly used by Katz and colleagues, and next it was developed by Willner [25]. CUMS is now the most useful model of depression. It is widely adapted rodent paradigm, used to induce depressive and anxiety-like behaviors. It imitates stressful situations of peoples' everyday life. Impulses that initiate the stress response in laboratory animals, so-called stressors, act usually from two to four weeks, and are potentially harmless to the body. Stressors used in the CUMS model include, among others, food and water reduction, swimming in hot and cold water, wet litter, squeezing the tail, temperature changes, sounds of predators, cage tilt of $45^{\circ}$, changes in light-dark cycle, the presence of rat droppings in the mouse cages etc. [5] (see also: Table 1).

Table 1. Stressor used during the CUMS model

\begin{tabular}{|c|c|}
\hline Forced swimming in cold water $\left(4^{\circ} \mathrm{C}\right)$ & $1,6,12,13,14,22,26,30$ \\
\hline Swimming in $45^{\circ} \mathrm{C}$ hot water & 26,30 \\
\hline Deprivation of water and food for $24 \mathrm{~h}$ & $3,6,12,13,14,20,21,22,26,29,30$ \\
\hline Noise & $6,12,20$ \\
\hline Intermittent white noise & 8,22 \\
\hline Tail squeezing ( $2 \mathrm{~min}$ ) & $6,13,14,30$ \\
\hline Inversion of the light/dark cycle & $3,6,7,15,26,30$ \\
\hline Cage shaking (30 $\mathrm{min})$ & 26,30 \\
\hline Cage tilting ( 12 or $15 \mathrm{~h}$ ) & $5,7,12,13,20,22,26$ \\
\hline Damp sawdust (12 or $15 \mathrm{~h}$ ) & $3,7,15,20$ \\
\hline Confinement in a tube $(3 \mathrm{~h})$ & 20 \\
\hline $\begin{array}{l}\text { Testing the dark phase and reversed light- } \\
\text { dark cycle }\end{array}$ & 7,20 \\
\hline Predator sounds & 15 \\
\hline Placement in an empty cage & 15 \\
\hline $\begin{array}{c}\text { Placement in an empty cage with water on } \\
\text { the bottom }\end{array}$ & 15 \\
\hline Switching cages & 15 \\
\hline Without sawdust + cat feces & 7 \\
\hline Without sawdust + cage tilting & 7 \\
\hline Wet bedding & $7,26,31$ \\
\hline Soiled bedding & 8 \\
\hline Rat droppings to mouse cages & 7,29 \\
\hline Overnight illumination & $8,12,13,14,22$ \\
\hline Physical restraint & $6,13,14,22$ \\
\hline Soiled cage & 12,22 \\
\hline Fasting for $48 \mathrm{~h}$ & 31 \\
\hline Empty water bottles & 22 \\
\hline Grouped housing & 22 \\
\hline Stroboscope lighting & 22 \\
\hline Restricted access to food & 22 \\
\hline $\begin{array}{c}\text { Exposure to a foreign object (e.g., a piece } \\
\text { of plastic) }\end{array}$ & 14 \\
\hline Small temperature reductions & 29 \\
\hline $24 \mathrm{~h}$ social isolation & 26 \\
\hline $24 \mathrm{~h}$ social crowding & 26 \\
\hline Hot stress in oven at $42^{\circ} \mathrm{C}$ & 26 \\
\hline
\end{tabular}

Furthermore, long-term exposure (of experimental animals) to various mild stressors is associated with significant changes in the behaviors. Three or four-weeks-exposure to stressors cause an increased level of corticosteroids in plasma, and anhedonia evaluated as reduction of saccharose preference in tested animals. A very important issue in this model is that all behavioral changes induced by stress can be reversed by giving antidepressants [20].

Besides above-mentioned animal models of depression, one of the most widely used screening tests for antidepressants is the forced swimming test (FST). Rodents are placed individually in glass cylinders and are allowed to swim for $6 \mathrm{~min}$. Total duration of immobility is recorded during the last 4 minutes of the test. The duration of immobility is defined as the time when an animal remained floating passively, made no attempts to escape and showed only slow movements to keep its head above the water.

Another widely used behavioral test used to study the effect of antidepressants is the tail suspension test (TST). In this paradigm, the rodents are suspended on the rod by tail for 6 minutes, and the duration of their immobility is recorded [7]. However, in these tests, a depression state is not induced, in contrary to CUMS model when exposure to various chronic stressors induces numerous changes leading consequently to depression state of the animals [28].

\section{CUMS procedure: animals, modifications, type of stressors}

CUMS stress procedure is currently the subject of numerous scientific research studies and still undergoes many modifications. The possible modification involves chronic exposure of laboratory animals (mice, rats) to different, randomly scheduled social and environmental stressors. The duration of each stressor action usually ranges up to several weeks (about 3-4 weeks) [6]. The use of the CUMS model allows explaining the pathophysiological mechanisms of depression, such as loss of neurogenesis and changes in the hypothalamic-pituitary-adrenal axis (HPA). This model is currently used in order to investigate

Table 2. Animals used in the CUMS model

\begin{tabular}{|c|c|}
\hline \multicolumn{2}{|l|}{ Gender } \\
\hline Rats & $1,2,3,6,8,12,13,14,22,29,30$ \\
\hline Mice & $5,7,10,15,20,21,23,27,29$ \\
\hline \multicolumn{2}{|l|}{ Sex } \\
\hline Males & $\begin{array}{c}1,2,3,5,6,7,8,10,12,13,14,20 \\
21,28,29,30,32\end{array}$ \\
\hline Females & 26 \\
\hline Both males and females & 15,23 \\
\hline \multicolumn{2}{|l|}{ Age } \\
\hline \begin{tabular}{|c|}
$\begin{array}{c}\text { Immature, i.e., } 20-23 \text { days (weanling), } 29-40 \\
\text { days (juvenile), days (young adulthood) old } \\
\text { and more than } 50 \text { days }\end{array}$ \\
\end{tabular} & $1,8,14$ \\
\hline Animals 2-4 months old & $7,10,15,20,27,31$ \\
\hline $\begin{array}{l}\text { Aged, i.e., 7-9 months and 24-25 months } \\
\text { old }\end{array}$ & 2 \\
\hline \multicolumn{2}{|l|}{ Housing } \\
\hline Individual cage & $1,20,21,30$ \\
\hline $\begin{array}{l}\text { In groups } \\
\text { (2-9/cage) }\end{array}$ & $\begin{array}{c}2,3,5,6,7,8,10,14,15,17,23 \\
26,30\end{array}$ \\
\hline \multicolumn{2}{|l|}{ Feeding } \\
\hline Access restricted (25-30 g/day) & 1,8 \\
\hline Ad libitum & $\begin{array}{c}2,3,5,6,7,8,10,12,13,15,20,21, \\
22,23,27,31\end{array}$ \\
\hline
\end{tabular}


the influence of environmental stressors, on the prodepresive behavior of tested laboratory animals, rats or mice [15].

Chronic stress procedure was initially developed in rats by Willner [25], and at present both rats and mice are commonly used (see also Table 2). All conditions, under which the research is performed, must be adapted to the species, sex, age, and physical state of the tested laboratory animals. These conditions should be especially important in the tests and models when a state of depression or anhedonia is provoked and can be manifested by behavioral disturbances [21].

\section{Neuronal processes and brain structures involved in the CUMS model}

Until recently, many of undertaken studies have indicated that depression occurs due to the numerous changes in the body, which may result from the reduction of structural plasticity of neurons [27]. CUMS performed on rodents is often used to explain the pathophysiology of nerve inflammation in depression and to assess disorders associated with an increased risk of neurodegenerative diseases [5]. Clinical and pre-clinical data show that depression is associated with activation of the immune system, which is manifested as the inflammation state. In particular, this disease is characterized by an increase in pro-inflammatory cytokines such as tumor necrosis factor (TNF) and interleukin-6. Depression may also be caused by an increase in peripheral cytokine. The drug therapy involves administration of their antagonists or antidepressants, also supporting the anti-inflammatory properties of cytokines [18].

Chronic exposure to the stress in the CUMS model has a large influence on the function of the brain regions involved in memory and learning process in rodents. Observed dysfunctions are accompanied by disturbances in the hormonal system of HPA and numerous changes in the complex cascades of intracellular processes involving $\mathrm{G}$ proteins, protein kinases, second messengers, and transcription factors. Many studies have shown a link between disorders caused by various long-term acting stress factors and depression.

Chronic stress causes a series of physiological changes in a body. One of the most important is the activation of the HPA, which is associated with excessive release of cortisol (called "stress hormone") in the blood. Moreover, increased glucocorticosteroids(GKS) level, induces damage to the dopaminergic, serotoninergic or glutamatergic neurons. GKS also cause the reduction in dendrites branching and reduction in the number of dendritic spines. Increased GKS blood levels also lead to inhibition of neurogenesis process. The consequence of all these changes is reduction in size of the hippocampus and the frontal cortex, which is characteristic for patients with severe depression [3].

Disorder of the HPA is one of the most important mechanisms of depression. Moreover, increased release of corticotropin $(\mathrm{CRH})$, caused by adrenocorticotropic hormone $(\mathrm{ACTH})$ releasing factor (CRF) is observed. CRF plays a very important role in the body's response to various stress stimuli, by enhancement of CRH and cortisol secretion. Moreover, CRH has its own very strong psychotropic effects (anxiety- or depressive-like reaction, sleeping and eating disorders). Chronic stress causes the enhanced CRH secretion as a result of the protein kinases phosphorylation and of CREB-transcription factor activation. $\mathrm{CRH}$ acts on receptors in the anterior pituitary and causes stimulation of ACTH release. Furthermore, ACTH increases the synthesis and release of adrenal GKS (e.g., cortisol). In chronic depression, two opposing processes occur simultaneously, i.e., stimulation of ACTH secretion by excessively secreted $\mathrm{CRH}$ and $\mathrm{ACTH}$-induced strong inhibition due to increased release of cortisol [19]. The results of a recent study clearly show that adding an additional gene coding $\mathrm{CRH}$ to the mouse genome, significantly increases anxiety reactions, and also enhances responses to stress. The influence of CRH on anxiety processes runs through the cell receptor CRHR 1 in the pituitary and other brain structures, such as the limbic pathway and the frontal cortex.

Direct mechanism of corticotropin action is not fully understood, because of interaction with multiple neurohormonal and neurotransmitter systems. Many studies confirmed the link between $\mathrm{CRH}$ and monoaminergic neurotransmitter, i.e., 5-hydroxy tryptamine (5-HT, serotonin) and noradrenaline (NA). This fact is confirmed by a significant influence of $\mathrm{CRH}$ on the tyrosine kinase activity participating in the NA synthesis. Appetite is associated with the $\mathrm{CRH}$ interactions with some endogenous substances that regulate appetite, like leptin.

The hippocampus and frontal cortex i.e., structures responsible for emotional responses, are particularly susceptible to this factor. Studies conducted on laboratory animals in CUMS model have shown that long-acting stressors cause atrophy of hippocampal pyramidal cells of CA3 and lower the resistance to other damaging agents (e.g., hypoglycemia and hypoxia). Chronic stress also impairs the neurogenesis that occurs in the hippocampus and the associative cortex. Thus, the neurogenesis is inhibited and in consequence hippocampus (structure responsible for emotional responses) size undergoes reduction [4].

\section{Drugs evaluated in the CUMS model}

CUMS has been used to assess the mechanism of action of some antidepressants and their effect on the HPA system. Particularly, from pharmacological point of view they have a significant effect on the nervous system, which protects neurons against a variety of stressful stimuli and against all kinds of interference of internal processes in the body. The goal of research studies carried out in this area is to prove neuroprotective properties of the psychotropic drugs [16]. Recently, a similar effect has been observed using four antidepressants in the mice CUMS model. The effect of imipramine $(20 \mathrm{mg} / \mathrm{kg})$, desipramine $(10 \mathrm{mg} / \mathrm{kg})$, maprotiline $(20$ $\mathrm{mg} / \mathrm{kg})$ and fluoxetine $(10 \mathrm{mg} / \mathrm{kg})$ in 6 -week schedule of administration was measured [28]. Results confirm the antidepressant and neuroprotective effects of above-mentioned drugs in ahedonia-related state in rodents.

A recent study on resveratrol, a natural polyphenol, aims at determining whether its chronic administration $(80 \mathrm{mg} /$ $\mathrm{kg}$, for 5 weeks) can affect cognitive deficits induced by chronic stress in laboratory animals and finding the possible mechanism of this phenomenon. The results of these studies show that administration of resveratrol causes a significant 
reduction in behavioral and biochemical changes in the brain caused by stress. These studies may indicate that resveratrol can have some neuroprotective properties and may be an effective therapeutic agent in the treatment of depression. This compound also participates in regulating the cortisol level in the blood, a neurotrophic factor BDNF in the prefrontal cortex as well as hippocampus and is responsible for the decrease in phosphorylation of cellular signal-regulated kinase [12].

The aim of another research was to investigate the mechanisms underlying the antidepressant effect of curcumin in the rat CUMS model. This study provides new information concerning the basic mechanisms leading to neuronal dysfunction in depression and the discovery of the therapeutic potential for the use of curcumin in clinical trials [33].

Results of many research studies have shown that the level of pro-inflammatory cytokines and oxidative stress (i.e., lipid peroxidation growth, reduced glutathione, and endogenous levels of oxidative enzymes) are increased in patients with depression. Therefore, the effect of sesamol, a strong antioxidant and inhibitor of cytokine production, was examined on biochemical and behavioral changes induced by chronic stress in mice. The chronic administration of sesamol (21-day) reverses the behavior caused by chronic stress (i.e., increased duration of immobility, reduced preference for glucose) and biochemical changes (i.e., decreased levels of glutathione, superoxide dismutase and catalase activity, increased levels of lipid peroxidation) [10].

Additionally, behavioral and biochemical effects of piperine were studied in rats exposed to the CUMS. Significant changes in rats' behaviors, including decrease in saccharose intake and increased duration of immobility in the FST were observed. The results also revealed that piperine could change the concentration of 5-HT and BDNF in the hippocampus and frontal cortex of rats, which may indicate its potential antidepressant activity also suggesting that piperine administration to rats significantly suppressed both biochemical and behavioral changes induced by the CUMS [14].

A recent study examined antidepressant effects of magnolol from the bark of Magnolia, used in China in treatment of psychiatric disorders, including depression. In this study, the antidepressant action of magnolol was studied in rats. These data have revealed that this compound has the ability to increase the expression of BDNF and enhances the activity of the serotoninergic system. These effects confirm antidepressant activity of magnolol [11].

Research studies describing the influence of agmatine on the CUMS in mice as well as its biochemical and behavioral changes have also been performed. This compound has been shown to exert modulatory action at multiple molecular targets, notably neurotransmitter systems, key ion channels, nitric oxide (NO) synthesis and polyamine metabolism. The results reveal that the chronic administration of agmatine reduces CUMS parameters characteristic for depression behavior in tested animals, i.e., increase in saccharose preference or reduction of immobility time in the FST. It has been proved that the administration of agmatine normalizes the elevated level of corticosterone and prevents the changes in body weight of animals subjected to the CUMS model. The pharmacological effects of agmatine were compared to the action of the selective serotonin reuptake inhibitor (SSRI) - fluoxetine. Results of this study clearly showed the antidepressant action of agmatine [23].

Recent studies using the CUMS model have also confirmed the action of Xiaochaihutang (XCHT), a substance used in China for thousands years for treatment of symptoms similar to depression. The results have confirmed that XCHT significantly increases food intake and saccharose preference in rats, and reduces all depression-like behavior. Moreover, XCHT significantly increases 5-HT and dopamine levels and improves cerebral expression of all factors (including BDNF), which were reduced in rats expressing symptoms of anhedonia. Therefore, these studies suggest that XCHT may have therapeutic effect in the treatment of depression and any related symptoms [22].

Another study aimed at demonstrating the antidepressant action of Fuzi polysaccharide-1 (FSP), a new water-soluble polysaccharide used for centuries in traditional Chinese medicine in the treatment of mood disorders. Research has shown that FSP increases the number of new cells in the dentate gyrus of adult mice, where the majority of the cells are differentiated into new neurons. It has been proved that the FSP reduces the duration of immobility in the FST and reverses the inhibition of neurogenesis process, characteristic for depression. Results of this study have revealed that acute treatment with FSP do not directly affect the level of monoamines in the brain, but considerably increases the level of BDNF in the hippocampus. The described study was designed to determine the utility of FSP as an antidepressant with a very fast onset of action, in contrast to many currently used anti-depressant drugs the therapeutic effect of which appears after several weeks of administration [29].

\section{CONCLUSION}

CUMS animal model is now a valuable tool to investigate the neurobiological, behavioral and hormonal changes underlying the psychopathology associated with stress. It plays a very important role in understanding the pathophysiological mechanisms of depression and efficacy of antidepressant therapy [15].

So far, many studies have confirmed that CUMS contributed to the exploration of many pathophysiological mechanisms of depression, such as inhibition of neurogenesis in the hippocampus, a disorder of the HPA axis, peripheral changes in the level of pro-inflammatory cytokines, increased lipid peroxidation, reduced glutathione level, increased levels of endogenous oxidative enzymes, and numerous nerve inflammations [13].

The purpose of CUMS animal model is therefore to determine the relation between the behavioral changes caused by stressful situations which tested animals are subjected to, and the identification of the clinical symptoms of depression [5]. 


\section{REFERENCES}

1. Bravo L. et al.: Pain exacerbates chronic mild stress-induced changes in noradrenergic transmission in rats. Eur. Neuropsychopharmacol., 24 (6), 996-1003, 2013.

2. Crema L.M. et al.: The effect of unpredictable chronic mild stress on depressive-like behavior and on hippocampal A1 and striatal A2A adenosine receptors. Physiol. Behav., 109, 1-7, 2013.

3. de Andrade J.S. et al.: Chronic unpredictable mild stress alters an anxiety-related defensive response, Fos immunoreactivity and hippocampal adult neurogenesis. Behav. Brain Res., 1, 250, 81-90, 2013.

4. Drevets W.C.: Neuroimaging and neuropathological studies of depression: implications for the cognitive-emotional features of mood disorders. Curr. Opin. Neurobiol., 11 (2), 240-249, 2001.

5. Farooq R.K. et al.: Is unpredictable chronic mild stress (UCMS) a reliable model to study depression-induced neuroinflammation? Behav. Brain Res., 23, 130-137, 2012.

6. Guan S. Z. et al.: Chronic unpredictable mild stress impairs erythrocyte immune function and changes T-lymphocyte subsets in a rat model of stress-induced depression. Environ. Toxicol. Pharmacol., 37, 414-422, 2014.

7. Ibarguen-Vargas Y. et al.: Deficit in BDNF does not increase vulnerability to stress but dampens antidepressant-like effects in the unpredictable chronic mild stress. Behav. Brain Res., 202, 245-251, 2009.

8. Javelot H. et al.: Behavioral and neurochemical effects of dietary methyl donor deficiency combined with unpredictable chronic mild stress in rats. Behav. Brain Res. 261, 8-16, 2014.

9. Krishnan V., Nestler EJ.: The molecular neurobiology of depression. Nature, 455, 894-902, 2008.

10. Kumar B., Kuhad A., Chopra K.: Neuropsychopharmacological effect of sesamol in unpredictable chronic mild stress model of depression: behavioral and biochemical evidences. Psychopharmacology, 214 (4), 819-828, 2011.

11. Li L.F.: Antidepressant-like effect of magnolol on BDNF up-regulation and serotonergic system activity in unpredictable chronic mild stress treated rats. Phytother. Res. 26 (8), 1189-1194, 2012.

12. Liu D. et al.: Resveratrol prevents impaired cognition induced by chronic unpredictable mild stress in rats. Prog. Neuropsychopharmacol. Biol. Psychiatry, 49, 21-29, 2013.

13. Luo J. et al.: Neotrofin reverses the effects of chronic unpredictable mild stress on behavior via regulating BDNF, PSD-95 and synaptophysin expression in rat. Behav. Brain Res., 253, 48-53, 2013.

14. Mao Q.Q. et al.: Piperine reverses chronic unpredictable mild stressinduced behavioral and biochemical alterations in rats. Cell Mol. Neurobiol., 34 (3), 403-408, 2014.

15. Mineur Y.S., Belzung C., Crusio W.E.: Effects of unpredictable chronic mild stress on anxiety and depression like behavior in mice. Behav. Brain Res., 175, 43-50, 2006.

16. Nestler E.J. et al.: Neurobiology of depression. Neuron, 34, 13-25, 2002.
17. Nollette C.: Learned helplessness. JEMS, 39 (2), 73, 2014.

18. O'Brien S.M., Scott L.V., Dinan T.G.: Cytokines: abnormalities in major depression and implications for pharmacological treatment. Hum. Psychopharmacol., 19 (6), 397-403, 2004.

19. Parker K.J., Schatzberg A.F., Lyons D.M.: Neuroendocrine aspects of hypercortisolism in major depression. Horm. Behav., 43 (1), 60-66, 2003.

20. Pothion S. et al.: Strain differences in sucrose preference and in the consequences of unpredictable chronic mild stress. Behav. Brain Res., 155, 135-146, 2004

21. Schweizer M.C., Henniger M.S., Sillaber I.: Chronic mild stress (CMS) in mice: of anhedonia, 'anomalous anxiolysis' and activity. PLoS One, 4 (1), e4326, 2009.

22. Su G.Y. et al.: Antidepressant-like effects of Xiaochaihutang in a rat model of chronic unpredictable mild stress. J Ethnopharmacol., 152(1), 217-226, 2014.

23. Taksande B.G.: Agmatine attenuates chronic unpredictable mild stress induced behavioral alteration in mice. Eur. J. Pharmacol., 720 (1-3), 115-120, 2013

24. Vollmayr B., Mahlstedt M.M., Henn F.A.: Neurogenesis and depression: what animal models tell us about the link. Eur. Arch. Psychiatry Clin. Neurosci., 257 (5), 300-303, 2007.

25. Willner P., Muscat R., Papp M.: Chronic mild stress-induced anhedonia: a realistic animal model of depression. Neurosci. Biobehav. Rev., 16, 525-534, 1992.

26. Wu L.M. et al.: Chronic unpredictable stress decreases expression of brain-derived neurotrophic factor (BDNF) in mouse ovaries: Relationship to oocytes developmental potential. PLoS One, 7 (12), e52331, 2012.

27. Yalcin I., Aksu F., Belzung C.: Effects of desipramine and tramadol in a chronic mild stress model in mice are altered by yohimbine but not by pindolol. Eur. J. Pharmacol., 514, 165-74, 2005.

28. Yalcin I., Belzung C., Surget A.: Mouse strain differences in the unpredictable chronic mild stress: a four-antidepressant survey. Behav. Brain Res., 193(1), 140-143, 2008.

29. Yan H.C. et al.: Fuzi polysaccharide-1 produces antidepressant-like effects in mice. Int. J. Neuropsychopharmacol., 13 (5), 623-633, 2010.

30. Yang C.R.: Foraging activity is reduced in a mouse model of depression. Neurotox. Res., 25 (3), 235-247, 2013.

31. Yang D. et al.: Reduced neurogenesis and pre-synaptic dysfunction in the olfactory bulb of a rat model of depression. Neuroscience, 192, 609-618, 2011.

32. Ye Y. et al.: Brain-derived neurotrophic factor (BDNF) infusion restored astrocytic plasticity in the hippocampus of a rat model of depression. Neurosci. Lett. 503, 15-19, 2011.

33. Zhang L. et al.: Effects of curcumin on chronic, unpredictable, mild, stress-induced depressive-like behaviour and structural plasticity in the lateral amygdala of rats. Int. J. Neuropsychopharmacol., 17(5), 793-806, 2014. 